\title{
On the Asymptotic Behavior and Radial Symmetry of Positive Solutions of Semilinear Elliptic Equations in $\boldsymbol{R}^{n}$ II. Radial Symmetry
}

\author{
YI LI \& WeI-MiNG NI
}

The main purpose of this paper is to prove Theorems 1 and 2 of the preceding paper, Part I, together with their extensions and related symmetry results. To make this part essentially self-contained, we shall apply the method developed in Section 2 to equations with radial symmetry. Combining the asymptotic behavior and the "moving plane" technique, we are then able to obtain the desired results.

Throughout this part of the paper we shall consider, for simplicity, bounded positive solutions of

$$
\Delta u+K(|x|) f(u)=0
$$

in $\boldsymbol{R}^{n}, n \geqq 3$. For the convenience of the reader, we list all the hypotheses on $K$ and $f$ that we shall need from time to time although perhaps not always simultaneously.

(K1) $K \geqq 0$ in $\boldsymbol{R}^{n}, K \in C^{\alpha}\left(\boldsymbol{R}^{n}\right)$ for some $\alpha>0$ and $K(|x|)=O\left(|x|^{-\tau}\right)$ near $x=\infty$ for some $\tau \geqq 0$.

(K2) $K(r)$ is nonincreasing in $r>0$.

(K3) There exists a constant $C$ such that

$$
|K(|x|)-K(|y|)| \leqq C|| x|-| y||\left[|x|^{-\tau}|y|^{-1}+|x|^{-1}|y|^{-\tau}\right]
$$

for all $x, y \in \boldsymbol{R}^{n}$ with $|x|,|y|$ large.

(f1) $f(u)>0$ if $u>0$, and $f(u)$ is Lipschitz continuous for $u \geqq 0$.

(f2) $f(u)=O\left(u^{p}\right)$ near $u=0$ for some $p \geqq 0$.

(f3) There exists a constant $C$ such that

$$
|f(u)-f(v)| \leqq C|u-v|\left(|u|^{p-1}+|v|^{p-1}\right)
$$

for all $u, v$ small and positive. 
We should remark that in case $K$ is in $C^{1}$, then (K3) is equivalent to $\left|K^{\prime}(t)\right| \leqq C t^{-(\tau+1)}$ for $t$ large. Similarly, if $f$ is in $C^{1}$, then (f3) is equivalent to $\left|f^{\prime}(t)\right| \leqq C t^{p-1}$ for $t>0$ and small.

\section{Asymptotic Behavior for Equations with Symmetry}

One key ingredient in proving our symmetry results is a more detailed asymptotic behavior of solutions of the equation

$$
\Delta u+K(|x|) f(u)=0 \quad \text { in } R^{n}, n \geqq 3 .
$$

Our first result deals with the zero limit case.

Theorem 3.2. Suppose that $(\mathrm{K} 1)$, (f1), (f2) and (f3) hold with $p>\max \left\{1, \frac{n-\tau}{n-2}\right\}$.
Let $u$ be a positive solution of (3.1) such that

$$
u(x)= \begin{cases}o\left(|x|^{\left.\min \left\{0, \frac{\tau-2}{p-2}\right\}\right)},\right. & \text { if } \tau \neq 2, \\ o\left((\log |x|)^{\frac{1}{p-1}}\right) & \text { if } \tau=2\end{cases}
$$

near $\infty$. Then

$$
u(x)=\frac{C_{1}}{|x|^{n-2}}+\frac{1}{|x|^{n-2}} W\left(\frac{1}{|x|}\right)+\frac{1}{|x|^{n-2}} R\left(\frac{x}{|x|^{2}}\right)
$$

near $\infty$, where

$$
W(r)=-\int_{0}^{r} \frac{1}{t^{n-1}} \int_{0}^{t} \varphi(s) s^{n-1} d s d t
$$

for some function $\varphi$ such that $\varphi(s)=O\left(s^{\gamma-2}\right)$ near $s=0$ with $\gamma=p(n-2)+\tau-n$, and $R$ is a Lipschitz function with $R(0)=0$.

Note that $W$ defined in (3.5) is the same as $W(\varphi)$ defined by (2.69).

Proof of Theorem 3.2. First, observe that $u(x)=O\left(|x|^{2-n}\right)$ near $\infty$. (This follows from Theorem 2.9 and the proofs of Theorems 2.25 and 2.31 in [LN1]. In fact, Theorems 2.25 and 2.31 in [LN1] are proved for radial solutions only, but their proofs work for general solutions after very minor modifications.) Then Theorem 2.4 in Part I applies and we may proceed as in the proof of Theorem 2.16. Setting

$$
y=\frac{x}{|x|^{2}}, \quad v(x)=|x|^{2-n} u(y)=|y|^{n-2} u(y)=C_{1}+v_{1}(x),
$$


where $v_{1}(x)=O\left(|x|^{\beta-\varepsilon}\right)$ near $x=0$ with $\beta=\min \{\gamma, 1\}$ for all $\varepsilon \in(0, \beta)$ and $C_{1}>0$ (by (2.6)), we have

$$
\begin{aligned}
0 & =\Delta v+|x|^{-n-2} K\left(\frac{1}{|x|}\right) f\left(|x|^{n-2} v\right) \\
& =\Delta v+|x|^{-n-2} K\left(\frac{1}{|x|}\right) f\left(|x|^{n-2} C_{1}\right)+f_{1}(x)
\end{aligned}
$$

where

$f_{1}(x)=|x|^{-n-2} K\left(\frac{1}{|x|}\right)\left[f\left(|x|^{n-2} C_{1}+|x|^{n-2} v_{1}\right)-f\left(|x|^{n-2} C_{1}\right)\right]=O\left(|x|^{\gamma-2+\beta-\varepsilon}\right)$ near $x=0$. Setting

$$
W_{1}(r)=-\int_{0}^{r} \frac{1}{t^{n-1}} \int_{0}^{t} s^{-n-2} K\left(\frac{1}{s}\right) f\left(s^{n-2} C_{1}\right) s^{n-1} d s d t
$$

we obtain from (3.7) that

$$
v(x)=C_{1}+W_{1}(|x|)+v_{2}(x)
$$

with $v_{2}(x)=O\left(|x|^{\min \{1, \gamma+\beta-\varepsilon\}}\right)$ near $x=0$. (The function $W_{1}$ defined here is clearly related to the $W_{1}$ defined in (2.70). This abuse of notation should not cause any confusion.) Noting that $W_{1}(|x|)=O\left(|x|^{\gamma}\right)$ near $x=0$ and that $\min \{1, \gamma+\beta-\varepsilon\} \geqq \beta$ if $\varepsilon$ is small, we have $v(x)=C_{1}+v_{1}(x)$ with $v_{1}(x)=$ $W_{1}(|x|)+v_{2}(x)=O\left(|x|^{\beta}\right)$ near $x=0$. Returning to (3.6) we see that the original estimate on $v_{1}$ is improved from $O\left(|x|^{\beta-\varepsilon}\right)$ to $O\left(|x|^{\beta}\right)$ near $x=0$. Repeating the above arguments, we again obtain (3.8) but with $v_{2}(x)=$ $O\left(|x|^{\min \{1, \gamma+\beta\}}\right)$ near $x=0$.

Now, substituting this (3.8) into (3.7) we deduce similarly that near $x=0$

$$
0=\Delta v+|x|^{-n-2} K\left(\frac{1}{|x|}\right) f\left(|x|^{n-2}\left[C_{1}+W_{1}(|x|)\right]\right)+f_{2}(x),
$$

where $f_{2}$ is defined by the last equality, and $f_{2}(x)=O\left(|x|^{\gamma-2+\min \{1, \gamma+\beta\}}\right)$ near $x=0$. Let $k$ be the integer such that $k y \leqq 1<(k+1) \gamma$. Then iterating the above argument $k$ times we arrive at

$$
0=\Delta v+|x|^{-n-2} K\left(\frac{1}{|x|}\right) f\left(|x|^{n-2}\left[C_{1}+W_{k}(|x|)\right]\right)+f_{k+1}(x)
$$

near $x=0$, where

near $x=0$, and

$$
f_{k+1}(x)=O\left(|x|^{\gamma-2+\min (1,(k+1) \beta 3}\right)=O\left(|x|^{\gamma-1}\right)
$$

$$
W_{l}(r)=-\int_{0}^{r} \frac{1}{t^{n-1}} \int_{0}^{t} s^{-n-2} K\left(\frac{1}{s}\right) f\left(s^{n-2}\left[C_{1}+W_{l-1}(s)\right]\right) s^{n-1} d s d t
$$


for $l=1,2, \ldots, k+1$ with $W_{0}(r) \equiv 0$. From (3.9) we conclude that

$$
v(x)=C_{1}+W_{k+1}(|x|)+R(x)
$$

where $R(x)$ is the sum of the Newtonian potential of $f_{k+1}$ and a harmonic function and $R(0)=0$. Thus $R$ is in $C^{1+\gamma}$ and in particular $R(x)=O(|x|)$ near $x=0 . \quad$ Q.E.D.

For the positive limit case we have to assume that $\tau>2$ in (K1) in view of Theorem 3.10 in $[\mathrm{N}]$, and then Theorem 2.32 in Part I applies. (The more general nonlinear term $f(u)$ causes no extra difficulties at all in the proof of Theorem 2.32.) Here we further divide the case into two subcases: $\tau>n$ and $n \geqq \tau>2$.

Theorem 3.12. Suppose that (K1), (f1) and (f3) hold with $\tau>n$ and that $u$ is a bounded positive solution of (3.1). Then $C_{0}=\lim _{x \rightarrow \infty} u(x)$ always exists. Moreover, if $C_{0}>0$, then

$$
u(x)=C_{0}+\frac{C_{1}}{|x|^{n-2}}+\frac{1}{|x|^{n-2}} W\left(\frac{1}{|x|}\right)+\frac{1}{|x|^{n-2}} R\left(\frac{x}{|x|^{2}}\right)
$$

near $x=\infty$ where

$$
W(r)=-\int_{0}^{r} \frac{1}{t^{n-1}} \int_{0}^{t} \varphi(s) s^{n-1} d s d t
$$

for some function $\varphi$ such that $\varphi(s)=O\left(s^{\tau-n-2}\right)$ near $s=0$, and $R$ is a Lipschitz function with $R(0)=0$.

Proof. The fact that $C_{0}=\lim _{x \rightarrow \infty} u(x)$ always exists follows from the proof of Theorem 2.32 (Lemma 2.8 in [LN1], to be precise). We now proceed as in the proof of Theorem 2.41. First, we set $\gamma=\tau-n$, and $u(x)=C_{0}+u_{1}(x)$ where

$$
u_{1}(x)=\frac{1}{n(n-2) \omega_{n}} \int_{\boldsymbol{R}^{n}} \frac{K(y) f(u(y))}{|x-y|^{n-2}} d y .
$$

Then the arguments used in the proof of Theorem 2.4 imply that

$$
\lim _{x \rightarrow \infty}|x|^{n-2} u_{1}(x)=\frac{1}{n(n-2) \omega_{n}} \int_{\mathbb{R}^{n}} K(y) f(u(y)) d y \equiv C_{1}>0 .
$$

Now letting

$$
y=\frac{x}{|x|^{2}}, \quad v(x)=|x|^{2-n} u_{1}(y)=C_{1}+v_{1}(x)
$$


where $v_{1}$ is defined by the last equality, we see that $v_{1}(x)=O\left(|x|^{\beta-\varepsilon}\right)$ near $x=0$, where $\beta=\min \{1, \gamma\}$ and $\varepsilon \in(0, \beta)$, again from the proof of Theorem 2.4. Then straightforward computation shows that

$$
\begin{aligned}
0 & =\Delta v+|x|^{-n-2} K\left(\frac{1}{|x|}\right) f\left(C_{0}+|x|^{n-2} v\right) \\
& =\Delta v+|x|^{-n-2} K\left(\frac{1}{|x|}\right) f\left(C_{0}+|x|^{n-2} C_{1}\right)+f_{1}(x)
\end{aligned}
$$

where

$$
\begin{aligned}
f_{1}(x) & =|x|^{-n-2} K\left(\frac{1}{|x|}\right)\left[f\left(C_{0}+|x|^{n-2}\left(C_{1}+v_{1}\right)\right)-f\left(C_{0}+|x|^{n-2} C_{1}\right)\right] \\
& =O\left(|x|^{\tau+\beta-4-\varepsilon}\right)
\end{aligned}
$$

near $x=0$. Noting that $\tau+\beta-4-\varepsilon>n-4+\beta+\varepsilon>-1$, we conclude similarly as before (see, e.g., the proof of Theorem 3.2) that

near $x=0$ where

$$
v(x)=C_{1}+W(|x|)+R(x)
$$

$$
W(r)=-\int_{0}^{r} \frac{1}{t^{n-1}} \int_{0}^{t} s^{-n-2} K\left(\frac{1}{s}\right) f\left(C_{0}+s^{n-2} C_{1}\right) s^{n-1} d s d t
$$

and $R$ is a $C^{1}$ function near $x=0$ with $R(0)=0$. Q.E.D.

Theorem 3.17. Suppose that (K1), (f1) and (f3) hold with $2<\tau \leqq n$ and that $u$ is a bounded positive solution of (3.1). Then $C_{0}=\lim _{x \rightarrow \infty} u(x)$ always exists. Moreover, if $C_{0}>0$, then

$$
u(x)=C_{0}+N_{f, k+1}(|x|)+\frac{c_{k+2}}{|x|^{n-2}}+\frac{1}{|x|^{n-2}} W\left(\frac{1}{|x|}\right)+\frac{1}{|x|^{n-2}} R\left(\frac{x}{|x|^{2}}\right)
$$

near $x=\infty$, where $k$ is the first integer such that $k(\tau-2)<n-2 \leqq$ $(k+1)(\tau-2)$ and $N_{f, k+1}$ is given by (2.93) in Part I, $R$ is Lipschitz continuous near 0 with $R(0)=0$ and

$$
W(r)=-\int_{0}^{r} \frac{1}{t^{n-1}} \int_{0}^{t} \varphi(s) s^{n-1} d s d t
$$

with $\varphi(s)=O\left(-s^{\tau-4} \log s\right)$ near $s=0$. 
Proof. We only consider the case $2<\tau<n$, because the case $\tau=n$ may be treated similarly. As in the proof of Theorem 2.94 or 2.75 (by the arguments leading to (2.64), (2.65)) we have

$$
u(x)=C_{0}+N_{f, k+1}(|x|)+u_{k+2}(x)
$$

near $x=\infty$, and

$$
u_{k+2}(x)-\frac{c_{k+2}}{|x|^{n-2}}=O\left(\frac{1}{|x|^{\delta+n-2}}\right) .
$$

near $x=\infty$ for all $\delta<\min \{1, \eta\}$ with $\eta=\tau-2$ and

$$
c_{k+2}=\frac{1}{n(n-2) \omega_{n}} \int_{R^{n}} K\left[f\left(C_{0}+N_{f, k}+u_{k+1}\right)-f\left(C_{0}+N_{f, k}\right)\right]
$$

where $N_{f, k}$ and $u_{k+1}$ satisfy the appropriate estimates between (2.59) and (2.64). (Note that the function $N_{f, l}$ defined by $(2.93)$ is radial since $K$ is radial, and that the constant $c_{k+2}$ here may be zero or negative. However, if we assume that $f$ is monotone, then $c_{k+2}$ is always positive as in Theorem 2.75.)

We now proceed in the usual manner. Setting

$$
v(x)=|x|^{2-n} u_{k+2}\left(\frac{x}{|x|^{2}}\right)=c_{k+2}+v_{1}(x)
$$

for $x$ near 0 , where $v_{1}$ is defined by the last equality, we have $v_{1}(x)=O\left(|x|^{\delta}\right)$ near $x=0$ for all $\delta<\min \{1, \eta\}$, and

$$
\begin{aligned}
& 0=\Delta v+|x|^{-n-2} K\left(\frac{1}{|x|}\right)\left\{f\left(C_{0}+N_{f, k+1}\left(\frac{1}{|x|}\right)+c_{k+2}|x|^{n-2}+|x|^{n-2} v_{1}(x)\right)\right. \\
& \left.-f\left(C_{0}+N_{f, k}\left(\frac{1}{|x|}\right)\right)\right\} \\
& =\Delta v+|x|^{-n-2} K\left(\frac{1}{|x|}\right)\left\{f\left(C_{0}+N_{f, k+1}\left(\frac{1}{|x|}\right)+c_{k+2}|x|^{n-2}\right)\right. \\
& \left.-f\left(C_{0}+N_{f, k}\left(\frac{1}{|x|}\right)\right)\right\}+f_{1}(x)
\end{aligned}
$$

where $f_{1}$ is defined by the last equality, and it follows from (2.61) that $f_{1}(x)=O\left(|x|^{\eta+\delta-2}\right)$ near $x=0$ for all $\delta<\min \{1, \eta\}$. Therefore we conclude similarly as before that

$$
v(x)=c_{k+2}+W_{1}(|x|)+v_{2}(x)
$$


near $x=0$ where $v_{2}(x)=O\left(|x|^{\min (\eta+\delta, 1\}}\right)$ near 0 and

$$
\begin{array}{r}
W_{1}(|x|)=-\int_{0}^{r} \frac{1}{t^{n-1}} \int_{0}^{t} s^{-n-2} K\left(\frac{1}{s}\right)\left\{f\left(C_{0}+N_{f, k+1}\left(\frac{1}{s}\right)+c_{k+2} s^{n-2}\right)\right. \\
\left.-f\left(C_{0}+N_{f, k}\left(\frac{1}{s}\right)\right)\right\} s^{n-1} d s d t .
\end{array}
$$

Iterating the above argument $h$ times where $h$ is the first positive integer such that $(h+1) \eta>1$, we arrive at

$$
\begin{array}{r}
0=\Delta v+|x|^{-n-2} K\left(\frac{1}{|x|}\right)\left\{f\left(C_{0}+N_{f, k+1}\left(\frac{1}{|x|}\right)+|x|^{n-2}\left(c_{k+2}+W_{h-1}(|x|)\right)\right)\right. \\
\left.-f\left(C_{0}+N_{f, k}\left(\frac{1}{|x|}\right)\right)\right\}+f_{h}(x)
\end{array}
$$

near $x=0$ with $f_{h}(x)=O\left(|x|^{h \eta+\delta-2}\right)$ near $x=0$ for all $\delta<\min \{1, \eta\}$ and $W_{l}, l=0,1,2, \ldots, h$, defined recursively as follows: $W_{0} \equiv 0$, and

$$
\begin{array}{r}
W_{l}(r)=-\int_{0}^{r} \frac{1}{t^{n-1}} \int_{0}^{t} s^{-n-2} K\left(\frac{1}{s}\right)\left\{f\left(C_{0}+N_{f, k+1}\left(\frac{1}{s}\right)+s^{n-2}\left(c_{k+2}+W_{l-1}(s)\right)\right)\right. \\
\left.-f\left(C_{0}+N_{f, k}\left(\frac{1}{s}\right)\right)\right\} s^{n-1} d s d t .
\end{array}
$$

Since $h \eta+\delta-2>-1$ if $\delta$ is sufficiently close to $\min \{1, \eta\}$, it follows that

$$
v(x)=c_{k+2}+W_{h}(|x|)+R(x)
$$

near $x=0$ where $R(x)$ is in $C^{1}$ and $R(0)=0$. It is easy to see from (2.61) that $W_{h}$ takes the form (3.19) with the desired estimates. Q.E.D.

\section{Some Technical Estimates}

In this section we collect a number of technical estimates which will be needed in proving our symmetry results in the next section.

Let $x=\left(x_{1}, x_{2}, \ldots, x_{n}\right)$ be a point in $\boldsymbol{R}^{n}$; we denote its reflection with respect to the hyperplane $T_{\lambda}=\left\{y=\left(y_{1}, \ldots, y_{n}\right) \in \boldsymbol{R}^{n} \mid y_{1}=\lambda\right\}$ by $x^{\lambda}$; i.e., $x^{\lambda}=\left(2 \lambda-x_{1}, x_{2}, \ldots, x_{n}\right)$. First observe that

$$
\left|x^{\lambda}\right|-|x|=\frac{4 \lambda\left(\lambda-x_{1}\right)}{|x|+\left|x^{\lambda}\right|}>0 \quad \text { if } \lambda>x_{1} \text { and } \lambda>0
$$


Lemma 4.2. Suppose that $\lambda>0, m \geqq 1$. Then for $x \in R^{n}$ with $|x|>2$ and $x_{1}<\lambda$ the following estimates hold:

$$
\begin{aligned}
\frac{4 \lambda\left(\lambda-x_{1}\right)}{|x|^{m}\left|x^{\lambda}\right|\left(|x|+\left|x^{\lambda}\right|\right)} \leqq \frac{1}{|x|^{m}}-\frac{1}{\left|x^{\lambda}\right|^{m}} \leqq \frac{4 m \lambda\left(\lambda-x_{1}\right)}{|x|^{m}\left|x^{\lambda}\right|\left(|x|+\left|x^{\lambda}\right|\right)}, \\
\left|\frac{x}{|x|^{m}}-\frac{x^{\lambda}}{\left|x^{\lambda}\right|^{m}}\right| \leqq\left(\frac{4 m \lambda\left(\lambda-x_{1}\right)}{|x|^{m-1}\left|x^{\lambda}\right|\left(|x|+\left|x^{\lambda}\right|\right)}+\frac{2\left(\lambda-x_{1}\right)}{\left|x^{\lambda}\right|^{m}}\right) .
\end{aligned}
$$

Moreover, if $R$ is Lipschitz continuous in the unit ball with $R(0)=0$, then

$$
\begin{aligned}
& \mid R\left(\frac{x}{|x|^{2}}\right) \frac{1}{|x|^{m}}-R\left(\frac{x^{\lambda}}{\left|x^{\lambda}\right|^{2}}\right) \frac{1}{\left|x^{\lambda}\right|^{m} \mid} \\
& \quad \leqq C(R)\left[\frac{4(m+2) \lambda\left(\lambda-x_{1}\right)}{|x|^{m+1}\left|x^{\lambda}\right|\left(|x|+\left|x^{\lambda}\right|\right)}+\frac{2\left(\lambda-x_{1}\right)}{\left|x^{\lambda}\right|^{m+2}}\right]
\end{aligned}
$$

where $C(R)$ is the Lipschitz constant for $R$ in the unit ball.

Proof. Since

$$
m b^{m-1}(b-a) \geqq b^{m}-a^{m} \geqq b^{m-1}(b-a)
$$

for $b \geqq a>0$, (4.3) follows immediately from (4.1). For (4.4) we have

$$
\begin{aligned}
\left|\frac{x}{|x|^{m}}-\frac{x^{\lambda}}{\left|x^{\lambda}\right|^{m}}\right| & \leqq\left|\frac{x}{|x|^{m}}-\frac{x}{\left|x^{\lambda}\right|^{m}}\right|+\left|\frac{x}{\left|x^{\lambda}\right|^{m}}-\frac{x^{\lambda}}{\left|x^{\lambda}\right|^{m}}\right| \\
& =|x|\left|\frac{1}{|x|^{m}}-\frac{1}{\left|x^{\lambda}\right|^{m}}\right|+\frac{\left|\left(x-x^{\lambda}\right)\right|}{\left|x^{\lambda}\right|^{m}}
\end{aligned}
$$

and then (4.3) applies. Finally using (4.3) and (4.4) we have

$$
\begin{aligned}
& \left|R\left(\frac{x}{|x|^{2}}\right) \frac{1}{|x|^{m}}-R\left(\frac{x^{\lambda}}{\left|x^{\lambda}\right|^{2}}\right) \frac{1}{\left|x^{\lambda}\right|^{m}}\right| \\
& \leqq\left|R\left(\frac{x}{|x|^{2}}\right) \frac{1}{|x|^{m}}-R\left(\frac{x}{|x|^{2}}\right) \frac{1}{\left|x^{\lambda}\right|^{m}}\right|+\left|R\left(\frac{x}{|x|^{2}}\right) \frac{1}{\left|x^{\lambda}\right|^{m}}-R\left(\frac{x^{\lambda}}{\left|x^{\lambda}\right|^{2}}\right) \frac{1}{\left|x^{\lambda}\right|^{m}}\right| \\
& =\left|R\left(\frac{x}{|x|^{2}}\right)\right| \cdot\left|\frac{1}{|x|^{m}}-\frac{1}{\left|x^{\lambda}\right|^{m}}\right|+\frac{1}{\left|x^{\lambda}\right|^{m}}\left|R\left(\frac{x}{|x|^{2}}\right)-R\left(\frac{x^{\lambda}}{\left|x^{\lambda}\right|^{2}}\right)\right|
\end{aligned}
$$

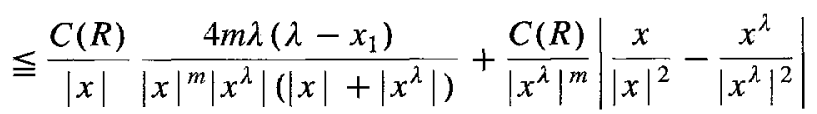

$$
\begin{aligned}
& \leqq C(R)\left[\frac{4 m \lambda\left(\lambda-x_{1}\right)}{|x|^{m+1}\left|x^{\lambda}\right|\left(|x|+\left|x^{\lambda}\right|\right)}+\frac{8 \lambda\left(\lambda-x_{1}\right)}{|x|\left|x^{\lambda}\right|^{m+1}\left(|x|+\left|x^{\lambda}\right|\right)}+\frac{2\left(\lambda-x_{1}\right)}{\left|x^{\lambda}\right|^{m+2}}\right] \text {, }
\end{aligned}
$$

and then (4.5) follows since $|x|<\left|x^{\lambda}\right|$. 
Lemma 4.6. Let $F(x)=O\left(|x|^{-q}\right)$ near $\infty$ for some $q>n$ and

$$
u(x)=\int_{\boldsymbol{R}^{n}} \frac{F(y)}{|x-y|^{n-2}} d y, \quad x \in \boldsymbol{R}^{n} .
$$

Suppose that

$$
\left(F(x)-F\left(x^{\lambda}\right)\right)\left(\lambda-x_{1}\right)=O\left(|x|^{-q}\right)
$$

near $\infty$ for every $\lambda$ sufficiently close to $\lambda_{0}$. If $\lambda_{i} \rightarrow \lambda_{0}$ and $\left\{x^{i}\right\}$ is a sequence of points in $\boldsymbol{R}^{n}$ such that $x^{i} \rightarrow \infty$ as $i \rightarrow \infty$ and $x_{1}^{i}<\lambda^{i}$, then

$$
\frac{\left|x^{i}\right|^{n}}{\lambda_{i}-x_{1}^{i}}\left(u\left(x^{i}\right)-u\left(x^{i \lambda_{i}}\right)\right) \rightarrow 2(n-2) \int_{\left[y_{1}<\lambda_{0}\right]}\left(F(y)-F\left(y^{\lambda_{0}}\right)\right)\left(\lambda_{0}-y_{1}\right) d y
$$

as $i \rightarrow \infty$.

Proof. We first observe that

$$
\begin{aligned}
u(x)-u\left(x^{\lambda}\right)= & \int_{\mathbb{R}^{n}}\left[\frac{f(y)}{|x-y|^{n-2}}-\frac{f(y)}{\left|x^{\lambda}-y\right|^{n-2}}\right] d y \\
= & \int_{\left[y_{1}<\lambda\right]} f(y)\left[\frac{1}{|x-y|^{n-2}}-\frac{1}{\left|x^{\lambda}-y\right|^{n-2}}\right] d y \\
& +\int_{\left[y_{1}>\lambda\right]} f(y)\left[\frac{1}{|x-y|^{n-2}}-\frac{1}{\left|x^{\lambda}-y\right|^{n-2}}\right] d y \\
= & \int_{\left[y_{1}<\lambda\right]}\left[f(y)-f\left(y^{\lambda}\right)\right]\left(\frac{1}{|x-y|^{n-2}}-\frac{1}{\left|x^{\lambda}-y\right|^{n-2}}\right) d y,
\end{aligned}
$$

where a change of variables from $y$ to $y^{\lambda}$ is used for the integral over the region $\left[y_{1}>\lambda\right]$. Next we note that as $i \rightarrow \infty$,

$$
\frac{\left|x^{i}\right|^{n}}{\lambda_{i}-x_{1}^{i}}\left(\frac{1}{\left|x^{i}-y\right|^{n-2}}-\frac{1}{\left|x^{i \lambda_{i}}-y\right|^{n-2}}\right) \rightarrow 2(n-2)\left(\lambda_{0}-y_{1}\right)
$$

uniformly on compact subsets of $\boldsymbol{R}^{n}$. Now the proof is similar to that of Lemma B in Part I (see Appendix A in pp. 394-397 in [GNN]) and therefore the details are omitted. Q.E.D.

Our next lemma is important and interesting in its own right.

Lemma 4.9. Let $F(x)=O\left(|x|^{-q}\right)$ near $\infty$ for some $q>n$ and let (4.7) hold near $\infty$ for every $\lambda \in R$. Then the function $I(\lambda)$ defined by

$$
I(\lambda)=\int_{\left[y_{1}<\lambda\right]}\left[F(y)-F\left(y^{\lambda}\right)\right]\left(\lambda-y_{1}\right) d y
$$


has the property that

$$
I(\lambda)-I\left(\lambda^{\prime}\right)=\left(\lambda-\lambda^{\prime}\right) \int_{R^{n}} F(y) d y
$$

for every $\lambda, \lambda^{\prime} \in \boldsymbol{R}$.

Proof. Let $r$ be a large number and $\lambda$ be fixed. Then

$$
\begin{aligned}
I(\lambda) & =\int_{\left[y_{1}<\lambda\right] \cap B_{r}(0)}\left(F(y)-F\left(y^{\lambda}\right)\right)\left(\lambda-y_{1}\right) d y+O\left(r^{n-q}\right) \\
& =\int_{\left[y_{1}<\lambda\right] \cap B_{r}(0)} F(y)\left(\lambda-y_{1}\right) d y+\int_{\left[y_{1}>\lambda\right] \cap B_{r}\left(0^{\lambda}\right)} F(y)\left(\lambda-y_{1}\right) d y+O\left(r^{n-q}\right) \\
& =\int_{B_{r}(0)} F(y)\left(\lambda-y_{1}\right) d y+\int_{S} F(y)\left(\lambda-y_{1}\right) d y+O\left(r^{n-q}\right)
\end{aligned}
$$

where $0^{\lambda}=(2 \lambda, 0, \ldots, 0)$ is the reflection of 0 with respect to $T_{\lambda}$ and

$$
S= \begin{cases}\left\{\left(\left[y_{1}<\lambda\right] \cap B_{r}(0)\right) \cup\left(\left[y_{1}>\lambda\right] \cap B_{r}\left(0^{\lambda}\right)\right)\right\} \backslash B_{r}(0) & \text { if } \lambda \geqq 0, \\ B_{r}(0) \backslash\left\{\left(\left[y_{1}<\lambda\right] \cap B_{r}(0)\right) \cup\left(\left[y_{1}>\lambda\right] \cap B_{r}\left(0^{\lambda}\right)\right)\right\} & \text { if } \lambda<0 .\end{cases}
$$

In case $\lambda \geqq 0$, then $|S| \leqq C\left[(r+2 \lambda)^{n}-r^{n}\right] \leqq C_{\lambda} r^{n-1}$ where the constant $C_{\lambda}$ depends on $\lambda$. Similarly $|S| \leqq C_{\lambda} r^{n-1}$ if $\lambda<0$. It then follows that

$$
\int_{S}\left|F(y)\left(\lambda-y_{1}\right)\right| d y \leqq C_{\lambda} r^{-q+1}|S|=O\left(r^{n-q}\right)
$$

for $r$ large. Thus

$$
I(\lambda)=\int_{B_{r}(0)} F(y)\left(\lambda-y_{1}\right) d y+O\left(r^{n-q}\right),
$$

which in turn implies that

$$
I(\lambda)-I\left(\lambda^{\prime}\right)=\int_{B_{r}(0)} F(y)\left(\lambda-\lambda^{\prime}\right) d y+O\left(r^{n-q}\right),
$$

and (4.11) follows immediately by letting $r \rightarrow \infty$ in (4.13). Q.E.D.

Remark 4.14. It follows from (4.11) (by setting $\lambda^{\prime}=0$ ) that $I(\lambda)=c \lambda+I(0)$ where $c=\int_{R^{n}} F(y) d y$. In particular, this implies that there exists a unique $\bar{\lambda}$ such that $I(\bar{\lambda})=0$ unless $I \equiv$ Constant (i.e., $\int_{R^{n}} F(y) d y=0$ ).

It will be necessary to estimate the function $W$ defined by (3.5), (3.14) or (3.19). Observe that they all take the form

$$
W(r)=-\int_{0}^{r} \frac{1}{t^{n-1}} \int_{0}^{t} \varphi(s) s^{n-1} d s d t
$$

where $\varphi(s)=O\left(s^{\beta-2}\right)$ near $s=0$ for some $0<\beta \leqq 1$. 
Lemma 4.16. Let $W$ be given by (4.15). Then

$$
|W(r)| \leqq C r^{\beta} \text { for } r \text { near } 0,
$$

$$
\left|W\left(\frac{1}{|x|}\right)-W\left(\frac{1}{\left|x^{\lambda}\right|}\right)\right| \leqq \frac{C\left|\lambda\left(\lambda-x_{1}\right)\right|\left(\max \left\{|x|,\left|x^{\lambda}\right|\right\}\right)^{1-\beta}}{|x|\left|x^{\lambda}\right|\left(|x|+\left|x^{\lambda}\right|\right)}
$$

for $|x|$ and $\left|x^{\lambda}\right|$ large.

Proof. Inequality (4.17) follows directly from (4.15). For (4.18) we compute

$$
\begin{aligned}
\left|W\left(\frac{1}{|x|}\right)-W\left(\frac{1}{\left|x^{\lambda}\right|}\right)\right| & =\left|\int_{1 /\left|x^{\lambda}\right|}^{1 /|x|} \frac{1}{t^{n-1}} \int_{0}^{t} \varphi(s) s^{n-1} d s d t\right| \leqq C\left|\int_{1 /\left|x^{\lambda}\right|}^{1 /|x|} t^{\beta-1} d t\right| \\
& \leqq C \max \left\{\left(\frac{1}{|x|}\right)^{\beta-1},\left(\frac{1}{\left|x^{\lambda}\right|}\right)^{\beta-1}\right\}\left|\frac{1}{|x|}-\frac{1}{\left|x^{\lambda}\right|}\right| .
\end{aligned}
$$

Our conclusion then follows from (4.3) and the assumption that $\beta \leqq 1$. Q.E.D.

Finally we come to the estimates of $N_{f, k+1}$ in Theorem 3.17. Recall that $\eta=\tau-2$ and

$$
N_{f, l}(x)=\frac{1}{n(n-2) \omega_{n}} \int_{\boldsymbol{R}^{n}} \frac{K(|y|) f\left(C_{0}+N_{f, l-1}(y)\right)}{|x-y|^{n-2}} d y, \quad l=1,2, \ldots
$$

with $N_{f, 0} \equiv 0$. Note that $N_{f, l}$ is radial for every $l$, as we pointed out in the proof of Theorem 3.17, and that we can virtually duplicate the proofs of (2.59) $-(2.61)$ to obtain

$$
\begin{aligned}
0 \leqq N_{f, l}(|x|) & \leqq C(1+|x|)^{-\eta}, \quad l=1,2, \ldots, k+1, \\
\mid N_{f, l}(|x|)-N_{f, l-1}(|x|) & \leqq C(1+|x|)^{-l \eta}, \quad l=1,2, \ldots, k, \\
\left|N_{f, k+1}(|x|)-N_{f, k}(|x|)\right| & \leqq C(1+|x|)^{2-n} \log (|x|+2),
\end{aligned}
$$

for all $x \in R^{n}$. It then follows from (4.19) that $\lim _{x \rightarrow \infty} N_{f, l}(|x|)=0$ and therefore

$$
N_{f, l}(|x|)=\int_{|x|}^{\infty} \frac{1}{t^{n-1}} \int_{0}^{t} K(s) f\left(C_{0}+N_{f, l-1}(s)\right) s^{n-1} d s d t
$$

The estimates we need are given by the next lemma.

Lemma 4.23. Suppose that (K1) and (f1) hold with $2<\tau \leqq n$. Suppose further that $K(r) \geqq C\left(1+r^{n}\right)^{-1}$ for all $r \geqq 0$ and for some constant $C>0$. Then for 
$x \in \boldsymbol{R}^{n} \backslash B_{2}(0)$ with $x_{1}<\lambda$ and $\lambda>0$,

$$
N_{f, l}(|x|)-N_{f, l}\left(\left|x^{\lambda}\right|\right) \geqq \frac{C \lambda\left(\lambda-x_{1}\right) \log |x|}{|x|^{n-2}\left|x^{\lambda}\right|\left(|x|+\left|x^{\lambda}\right|\right)}
$$

for $l=1,2, \ldots, k+1$.

Proof. Since $N_{f, l} \geqq 0$ and $f>0$ in $\boldsymbol{R}^{+}$, from (4.22) we have

$$
\begin{aligned}
N_{f, l}(|x|)-N_{f, l}\left(\left|x^{\lambda}\right|\right) & =\int_{|x|}^{\left|x^{\lambda}\right|} \frac{1}{t^{n-1}} \int_{0}^{t} K(s) f\left(C_{0}+N_{N, l-1}(s)\right) s^{n-1} d s d t \\
& \geqq C \int_{|x|}^{\left|x^{\lambda}\right|} \frac{1}{t^{n-1}} \int_{0}^{t} \frac{s^{n-1}}{1+s^{n}} d s d t \\
& =C \int_{|x|}^{\left|x^{\lambda}\right|} \frac{\log \left(1+t^{n}\right)}{t^{n-1}} d t \geqq C \log \left(1+|x|^{n}\right)\left(\frac{1}{|x|^{n-2}}-\frac{1}{\left|x^{\lambda}\right|^{n-2}}\right),
\end{aligned}
$$

and our conclusion (4.24) follows from (4.3). Q.E.D.

The last preliminary result we need is the following version of Hopf's Strong Maximum Principle and Boundary Point Lemma.

Lemma 4.25. Let $u$ be nonnegative and satisfy the inequality

$$
\triangle u+c(x) u \leqq 0 \quad \text { in } \Omega,
$$

where $\Omega$ is an open set in $\boldsymbol{R}^{n}$ with $\partial \Omega$ smooth and $c \in L_{\mathrm{loc}}^{\infty}(\bar{\Omega})$. Then either $u \equiv 0$ in $\Omega$ or $u(x)>0$ for all $x \in \Omega$. Moreover, if $u \neq 0$ in $\Omega$ and $u(\bar{x})=0$ for some point $\bar{x} \in \partial \Omega$, then $(\partial u / \partial v)(\bar{x})<0$ where $v$ is the unit outer normal of $\partial \Omega$ at $\bar{x}$.

The proof is standard; see for instance Chapter 3 of [GT].

\section{Symmetry}

We are now ready to prove our main results in this paper concerning radial symmetry of positive solutions of the equation

$$
\Delta u+K(|x|) f(u)=0
$$

in $\boldsymbol{R}^{n}, n \geqq 3$. We remark again that our method of proof does apply to more general equations (see Remark 5.18 at the end of this section). First, we treat 
the zero limit case. In case $K \equiv$ constant and $f$ is "nice", the following result improves Theorem 1 in [GNN].

Theorem 5.2. Suppose that (K1)-(K3) and (f1)-(f3) hold with $p>\max \left\{1, \frac{n-\tau}{n-2}\right\}$. Let $u$ be a positive solution of (5.1) such that

$$
u(x)= \begin{cases}o\left(|x|^{\left.\min \left\{o, \frac{\tau-2}{p-2}\right\}\right)}\right. & \text { if } \tau \neq 2, \\ o\left((\log |x|)^{-\frac{1}{p-1}}\right) & \text { if } \tau=2\end{cases}
$$

near $x=\infty$. Then $u$ must be radially symmetric about some point $z$ in $\boldsymbol{R}^{n}(z$ must be the origin if $K$ is not a constant) and $u_{r}<0$ for $r=|x-z|>0$. Furthermore,

$$
u(r)=\frac{C}{r^{n-2}}+O\left(\frac{1}{r^{n-2+\beta}}\right) \quad \text { near } r=\infty,
$$

where $\beta=\min \{1, \gamma\}$ and $\gamma=\tau+p(n-2)-n$.

Proof. In order to apply the moving plane and reflection device, we set

$$
\Lambda=\left\{\lambda \in \boldsymbol{R} \mid u(x)>u\left(x^{\lambda}\right) \text { for all } x \in \boldsymbol{R}^{n} \text { with } x_{1}<\lambda \text { and } \frac{\partial u}{\partial x_{1}}<0 \text { on } T_{\lambda}\right\} \text {. }
$$

(Recall that $T_{\lambda}$ is the hyperplane perpendicular to $x_{1}$-axis at $x_{1}=\lambda_{\text {. }}$ )

Step 1. $\lambda \in \Lambda$ for all sufficiently large $\lambda$.

Since $u>0$ in $\boldsymbol{R}^{n}$ and $u(x) \rightarrow 0$ as $x \rightarrow \infty$, we have that for every $r \geqq 2$, there exists $\lambda(r)>r$ such that if $\lambda \geqq \lambda(r)$, then $u(x)>u\left(x^{\lambda}\right)$ for all $|x| \leqq r$.

On the other hand, by Theorem 3.2, $u$ satisfies (3.4). Thus for $\lambda \geqq \lambda(r)$, $|x|>r \geqq 2$ and $x_{1}<\lambda$, we have, by (4.17), (4.18), (4.3) and (4.5), that

$$
\begin{gathered}
u(x)-u\left(x^{\lambda}\right)=\left(\frac{1}{|x|^{n-2}}-\frac{1}{\left|x^{\lambda}\right|^{n-2}}\right)\left[C_{1}+W\left(\frac{1}{|x|}\right)\right]+\frac{1}{\left|x^{\lambda}\right|^{n-2}}\left[W\left(\frac{1}{|x|}\right)\right. \\
\left.-W\left(\frac{1}{\left|x^{\lambda}\right|}\right)\right]+\frac{1}{|x|^{n-2}} R\left(\frac{x}{|x|^{2}}\right)-\frac{1}{\left|x^{\lambda}\right|^{n-2}} R\left(\frac{x^{\lambda}}{\left|x^{\lambda}\right|^{2}}\right) \\
\geqq \frac{4 \lambda\left(\lambda-x_{1}\right)}{|x|^{n-2}\left|x^{\lambda}\right|\left(|x|+\left|x^{\lambda}\right|\right)}\left[C_{1}-\frac{C}{|x|^{\beta}}\right]-\frac{C \lambda\left(\lambda-x_{1}\right)\left(\max \left\{|x|,\left|x^{\lambda}\right|\right\}\right)^{1-\beta}}{\left|x^{\lambda}\right|^{n-1}|x|\left(|x|+\left|x^{\lambda}\right|\right)} \\
\quad-C(R)\left[\frac{4 n \lambda\left(\lambda-x_{1}\right)}{|x|^{n-1}\left|x^{\lambda}\right|\left(|x|+\left|x^{\lambda}\right|\right)}+\frac{2\left(\lambda-x_{1}\right)}{\left.\left|x^{\lambda}\right|^{n}\right]}\right. \\
\geqq \frac{4 \lambda\left(\lambda-x_{1}\right)}{|x|^{n-2}\left|x^{\lambda}\right|\left(|x|+\left|x^{\lambda}\right|\right)}\left[C_{1}-\frac{C}{|x|^{\beta}}-\frac{n C(R)}{|x|}-\frac{C(R)}{\lambda}\right]
\end{gathered}
$$


since $|x|<\left|x^{\lambda}\right|$ and

$$
\begin{aligned}
\frac{\lambda\left(\lambda-x_{1}\right)\left|x^{\lambda}\right| 1-\beta}{\left|x^{\lambda}\right|^{n-1}|x|\left(|x|+\left|x^{\lambda}\right|\right)} & =\frac{\lambda\left(\lambda-x_{1}\right)}{|x|^{n-2}\left|x^{\lambda}\right|\left(|x|+\left|x^{\lambda}\right|\right)} \cdot \frac{|x|^{n-3}}{\left|x^{\lambda}\right|^{n-3+\beta}} \\
& \leqq \frac{\lambda\left(\lambda-x_{1}\right)}{|x|^{n-2}\left|x^{\lambda}\right|\left(|x|+\left|x^{\lambda}\right|\right)} \cdot \frac{1}{|x|^{\beta}} .
\end{aligned}
$$

Choosing $r$ so large that $C_{1}>C r^{-\beta}+(n+1) C(R) r^{-1}$, we see that $u(x)>u\left(x^{\lambda}\right)$ for all $\lambda \geqq \lambda(r)$ and for all $x$ with $x_{1}<\lambda$.

It remains to show that $\partial u / \partial x_{1}<0$ on $T_{\lambda}$ for all $\lambda \geqq \lambda(r)$. This follows easily from the Maximum Principle (Lemma 4.25). For $\lambda \geqq \lambda(r)$, in the region $\left\{x \in \boldsymbol{R}^{n} \mid x_{1}<\lambda\right\}$ we have $u(x)-u\left(x^{\lambda}\right)>0$, and

$$
\begin{aligned}
\Delta\left(u(x)-u\left(x^{\lambda}\right)\right)+K(|x|) \frac{f(u(x))-f\left(u\left(x^{\lambda}\right)\right)}{u(x)-u\left(x^{\lambda}\right)}\left(u(x)-u\left(x^{\lambda}\right)\right) & \\
= & \left(K\left(\left|x^{\lambda}\right|\right)-K(|x|)\right) f\left(u\left(x^{\lambda}\right)\right) \leqq 0
\end{aligned}
$$

since $|x|<\left|x^{\lambda}\right|$ and $K$ is monotone. Observe that $u(x)-u\left(x^{\lambda}\right)=0$ on $T_{\lambda}$; thus Lemma 4.25 implies that $\left(\partial / \partial x_{1}\right)\left(u(x)-u\left(x^{\lambda}\right)\right)<0$ on $T_{\lambda}$. Since $\left(\partial / \partial x_{1}\right) u\left(x^{\lambda}\right)=-\left(\partial / \partial x_{1}\right) u(x)$ for $x \in T_{\lambda}$, our assertion is established.

Step 2. Let $F(x)=K(|x|) f(u(x))$ for $x \in \boldsymbol{R}^{n}$ and for $\lambda \in \boldsymbol{R}$ let

$$
I(\lambda)=\int_{\left[x_{1}<\lambda\right]}\left[F(x)-F\left(x^{\lambda}\right)\right]\left(\lambda-x_{1}\right) d x .
$$

Then $I(\lambda)=C \lambda+I(0)$ where $C=\int_{R^{n}} F(y) d y>0$. In particular, there exists a unique $\bar{\lambda}$ such that $I(\bar{\lambda})=0$.

This follows immediately from Lemma 4.9 and Remark 4.14 provided that $F$ defined here satisfies the hypothesis of Lemma 4.9. Since $F(x)=$ $K(|x|) f(u(x))=O\left(|x|^{-\tau-p(n-2)}\right)$ near $\infty$ by Theorem 3.2, it remains to verify that $F$ satisfies (4.7). By (K1), (K3), (f3), (4.1), (4.3), Theorem 3.2 and a computation similar to (5.5), we obtain

$$
\begin{aligned}
\left|F(x)-F\left(x^{\lambda}\right)\right| \leqq & K(x)\left|f(u(x))-f\left(u\left(x^{\lambda}\right)\right)\right|+\left|K(x)-K\left(x^{\lambda}\right)\right| f\left(u\left(x^{\lambda}\right)\right) \\
\leqq & C|x|^{-\tau-(n-2)(p-1)}\left|u(x)-u\left(x^{\lambda}\right)\right| \\
& +C|| x|-| x^{\lambda}||\left(|x|^{-\tau}\left|x^{\lambda}\right|^{-1}+|x|^{-1}\left|x^{\lambda}\right|^{-\tau}\right)\left|x^{\lambda}\right|^{-p(n-2)} \\
\leqq & C \frac{\left|\lambda\left(\lambda-x_{1}\right)\right|}{|x|^{n-2}\left|x^{\lambda}\right|\left(|x|+\left|x^{\lambda}\right|\right)}|x|^{-\tau-(n-2)(p-1)} \\
& +C \frac{\left|\lambda\left(\lambda-x_{1}\right)\right|}{\left(|x|+\left|x^{\lambda}\right|\right)\left|x^{\lambda}\right|^{p(n-2)}}\left(\frac{1}{|x|^{\tau}\left|x^{\lambda}\right|}+\frac{1}{\left|x^{\lambda}\right|^{\tau}|x|}\right) \\
\leqq & C_{\lambda}|x|^{-\tau-p(n-2)-1}
\end{aligned}
$$


for $|x|$ large, where the constant $C_{\lambda}$ is bounded uniformly in $\lambda$ for $\lambda$ in a bounded set. Thus (4.7) holds for every $\lambda \in R$.

Step 3. If $K$ 三 constant, then $(0, \infty) \subseteq \Lambda$.

We first show that $\left(\bar{\lambda}^{\prime}, \infty\right) \cap A$ is open, where $\bar{\lambda}^{\prime}=\max \{\bar{\lambda}, 0\}$. Suppose that this is false, i.e., there exist a sequence $\left\{x^{i}\right\}$ in $\boldsymbol{R}^{n}$ and a sequence $\left\{\lambda_{i}\right\} \subseteq\left(\bar{\lambda}^{\prime}, \infty\right)$ such that $\lambda_{i} \rightarrow \hat{\lambda} \in\left(\bar{\lambda}^{\prime}, \infty\right) \cap \Lambda$ and $u\left(x^{i}\right) \leqq u\left(x^{i \lambda_{i}}\right)$ with $x_{1}^{i}<\lambda_{i}$. If $\left\{x^{i}\right\}$ has a subsequence, denoted also by $\left\{x^{i}\right\}$, which converges to $\bar{x}$, then $u(\bar{x}) \leqq u\left(\bar{x}^{\hat{\lambda}}\right)$ and $\bar{x}_{1} \leqq \hat{\lambda}$. Since $\hat{\lambda} \in A$, we must have $\bar{x}_{1}=\hat{\lambda}$ and $\left(\partial u / \partial x_{1}\right)(\bar{x}) \geqq 0$. On the other hand, in $x_{1}<\hat{\lambda}, K(|x|) \geqq K\left(\left|x^{\hat{\lambda}}\right|\right)$ since $\hat{\lambda}>0$, and the function $w(x)=u(x)-u\left(x^{\hat{\lambda}}\right)$ satisfies

$$
\begin{aligned}
& \Delta w+K(|x|) \frac{f(u(x))-f\left(u\left(x^{\hat{\lambda}}\right)\right)}{u(x)-u\left(x^{\hat{\lambda}}\right)} w=\left[K\left(\left|x^{\hat{\lambda}}\right|\right)-K(|x|)\right] f\left(u\left(x^{\hat{\lambda}}\right)\right) \leqq 0, \\
& w>0 \text { in } x_{1}<\hat{\lambda} \text { and } w=0 \text { on } x_{1}=\hat{\lambda} .
\end{aligned}
$$

But then Lemma 4.25 implies that $0>\left(\partial w / \partial x_{1}\right)(\bar{x})=2\left(\partial u / \partial x_{1}\right)(\bar{x})$, a contradiction. Therefore we must have $\left|x^{i}\right| \rightarrow \infty$ as $i \rightarrow \infty$. From the proof of Step 2 we see that Lemma 4.6 applies and we conclude from (4.8) that $I(\hat{\lambda}) \leqq 0$. This is again a contradiction since $\hat{\lambda}>\bar{\lambda}$ and $I(\hat{\lambda})=C(\hat{\lambda}-\bar{\lambda})>0$ by Step 2 . This proves that $\left(\bar{\lambda}^{\prime}, \infty\right) \cap A$ is open.

Next we assert that $\left(\bar{\lambda}^{\prime}, \infty\right) \subseteq \Lambda$. Suppose for contradiction that $(\tilde{\lambda}, \infty)$ is the unbounded component of $\left(\bar{\lambda}^{\prime}, \infty\right) \cap \Lambda$ with $\tilde{\lambda}>\bar{\lambda}^{\prime}$. (By Step 1 this unbounded component does exist). Then the continuity of $u$ guarantees that $u(x) \geqq u\left(x^{\tilde{\lambda}}\right)$ for all $x \in \boldsymbol{R}^{n}$ with $x_{1}<\tilde{\lambda}$. Moreover, in $x_{1}<\tilde{\lambda}, K(|x|) \geqq K\left(\left|x^{\tilde{\lambda}}\right|\right)$ since $\tilde{\lambda}>0$, and the function $\tilde{w}(x)=u(x) \div u\left(x^{\tilde{\lambda}}\right)$ satisfies

$$
\begin{aligned}
& \Delta \tilde{w}+K(|x|) \frac{f(u(x))-f\left(u\left(x^{\hat{\lambda}}\right)\right)}{u(x)-u\left(x^{\hat{\lambda}}\right)} \tilde{w}=\left[K\left(\left|x^{\tilde{\lambda}}\right|\right)-K(|x|)\right] f\left(u\left(x^{\tilde{\lambda}}\right)\right) \leqq 0, \\
& \tilde{w} \geqq 0 \text { for } x_{1}<\hat{\lambda} \text { and } \tilde{w}=0 \text { for } x_{1}=\tilde{\lambda} .
\end{aligned}
$$

Then Lemma 4.25 implies that either $u(x) \equiv u\left(x^{\tilde{\lambda}}\right)$ for $x_{1}<\tilde{\lambda}$ or $\tilde{\lambda} \in \Lambda$. Since $\tilde{\lambda} \notin \Lambda$ by our assumption, we must have that $u(x) \equiv u(\tilde{\lambda})$ for $x_{1}<\tilde{\lambda}$. This in turn implies that $K(|x|) \equiv K\left(\left|x^{\tilde{\lambda}}\right|\right)$ for $x_{1}<\tilde{\lambda}$ which is impossible since $K \neq$ constant. Thus our assertion holds.

Note that exactly the same arguments in the paragraph above applying to the function $u(x)-u\left(x^{\bar{\lambda}}\right)$ in $x_{1}<\bar{\lambda}$ yields that $\bar{\lambda} \in \Lambda$ if $\bar{\lambda}>0$. It then follows from Lemma $\left(H_{1}^{\prime \prime}\right)$ in [GNN; pp. 387, 388] that for some $\mu>0$

$$
u(x)-u\left(x^{\bar{\lambda}}\right) \geqq \mu\left(\bar{\lambda}-x_{1}\right)|x|^{-n}
$$

in $x_{1}<\bar{\lambda}$ with $|x|$ large (provided that $\bar{\lambda}>0$ ), since

$$
K(|x|) \frac{f(u(x))-f\left(u\left(x^{\bar{\lambda}}\right)\right)}{u(x)-u\left(x^{\bar{\lambda}}\right)}=O\left(|x|^{\tau+(p-1)(n-2)}\right)
$$

near $x=\infty$ (in view of (K1), (f3)) and since $\tau+(p-1)(n-2)>2$. 
To finish the proof of Step 3, we only have to show that $\bar{\lambda} \leqq 0$ since it would then follow that $\bar{\lambda}^{\prime}=0$. To this end we suppose that $\bar{\lambda}>0$ and we shall use (5.10) to derive a contradiction.

Observe that for every $\lambda \in(0, \bar{\lambda})$, there exists an $r_{\lambda}$ such that $u(x)<u\left(x^{\lambda}\right)$ for all $|x|>r_{\lambda}$ with $x_{1}<\lambda$. For otherwise there is a sequence $\left\{x^{i}\right\}$ with $u\left(x^{i}\right) \geqq u\left(x^{i \lambda}\right), x_{1}^{i}<\lambda$ and $\left|x^{i}\right| \rightarrow \infty$. Then Lemma 4.6 implies that $I(\lambda) \geqq 0$, which is not possible since $0=I(\bar{\lambda})>I(\lambda)$ by Step 2 .

For $0<\lambda<\bar{\lambda}$ we have, by (5.10) and Theorem 3.2, that

$$
\begin{aligned}
u(x)-u\left(x^{\lambda}\right)= & u(x)-u\left(x^{\bar{\lambda}}\right)+u\left(x^{\bar{\lambda}}\right)-u\left(x^{\lambda}\right) \\
\geqq & \frac{\mu\left(\bar{\lambda}-x_{1}\right)}{|x|^{n}}+\left(\frac{1}{\left|x^{\bar{\lambda}}\right|^{n-2}}-\frac{1}{\left|x^{\lambda}\right|^{n-2}}\right)\left[C_{1}+W\left(\frac{1}{\left|x^{\bar{\lambda}}\right|}\right)\right] \\
& +\frac{1}{\left|\overline{x^{\lambda}}\right|^{n-2}}\left[W\left(\frac{1}{\left|x^{\bar{\lambda}}\right|}\right)-W\left(\frac{1}{\left|x^{\lambda}\right|}\right)\right] \\
& +\frac{1}{\left|x^{\bar{\lambda}}\right|^{n-2}} R\left(\frac{x^{\bar{\lambda}}}{\left|x^{\bar{\lambda}}\right|^{2}}\right)-\frac{1}{\left|x^{\lambda}\right|^{n-2}} R\left(\frac{x^{\lambda}}{\left|x^{\lambda}\right|^{2}}\right)
\end{aligned}
$$

for $x_{1}<\lambda(<\bar{\lambda})$ and $|x|$ large, where $W$ and $R$ are given by Theorem 3.2. To estimate the various terms involved in (5.11), we proceed as in Section 4. As in the derivation of (4.3) we have, for $m \geqq 1$,

$$
\begin{aligned}
& \left|\frac{1}{\left|x^{\bar{\lambda}}\right|^{m}}-\frac{1}{\left|x^{\lambda}\right|^{m}}\right| \leqq C \frac{1}{\left|x^{\lambda}\right|^{m-1}}\left|\frac{1}{\left|x^{\bar{\lambda}}\right|}-\frac{1}{\left|x^{\bar{\lambda}}\right|}\right|=\frac{C(\bar{\lambda}-\lambda)\left(\bar{\lambda}+\lambda-x_{1}\right)}{\left|x^{\lambda}\right|^{m}\left|x^{\bar{\lambda}}\right|\left(\left|x^{\lambda}\right|+\left|x^{\bar{\lambda}}\right|\right)}, \\
& \left|\frac{1}{\left|x^{\lambda}\right|^{m}} R\left(\frac{x^{\lambda}}{\left|x^{\lambda}\right|^{2}}\right)-\frac{1}{\left|x^{\bar{\lambda}}\right|^{m}} R\left(\frac{x^{\bar{\lambda}}}{\left|x^{\bar{\lambda}}\right|^{2}}\right)\right| \\
& \leqq\left|R\left(\frac{x^{\lambda}}{\left|x^{\lambda}\right|^{2}}\right)\right|\left|\frac{1}{\left|x^{\lambda}\right|^{m}}-\frac{1}{\left|x^{\bar{\lambda}}\right|^{m}}\right|+\frac{1}{\left|x^{\bar{\lambda}}\right|^{m}}\left|R\left(\frac{x^{\lambda}}{\left|x^{\lambda}\right|^{2}}\right)-R\left(\frac{x^{\bar{\lambda}}}{\left|x^{\bar{\lambda}}\right|^{2}}\right)\right| \\
& \leqq \frac{C(\bar{\lambda}-\lambda)\left(\bar{\lambda}+\lambda-x_{1}\right)}{\left|x^{\lambda}\right|^{m+1}\left|x^{\bar{\lambda}}\right|\left(\left|x^{\lambda}\right|+\left|x^{\bar{\lambda}}\right|\right)}+\frac{C(\bar{\lambda}-\lambda)}{\left|x^{\bar{\lambda}}\right|^{m+2}}
\end{aligned}
$$

since $\left|x^{\lambda}\right|<\left|x^{\bar{\lambda}}\right|$. Also, as in the proof of (4.18), we deduce

$$
\left|W\left(\frac{1}{\left|x^{\lambda}\right|}\right)-W\left(\frac{1}{\left|x^{\bar{\lambda}}\right|}\right)\right| \leqq \frac{C(\bar{\lambda}-\lambda)\left(\bar{\lambda}+\lambda-x_{1}\right)}{\left|x^{\lambda}\right|\left|x^{\bar{\lambda}}\right|^{\beta}\left(\left|x^{\lambda}\right|+\left|x^{\bar{\lambda}}\right|\right)} .
$$

To simplify our estimates, we choose $|x|$ large with $x_{1}=0$ in (5.11)-(5.14) and obtain, by $|x| \leqq\left|x^{\lambda}\right| \leqq\left|x^{\bar{\lambda}}\right|$, that

$$
u(x)-u\left(x^{\lambda}\right) \geqq \frac{\mu \bar{\lambda}}{|x|^{n}}-\frac{C(\bar{\lambda}-\lambda)}{|x|^{n}}-\frac{C(\bar{\lambda}-\lambda)}{|x|^{n+\beta}}-\frac{C(\bar{\lambda}-\lambda)}{|x|^{n+1}},
$$


where the constant $C$ is independent $\lambda \in(0, \bar{\lambda})$. From (5.15) we conclude that if $\bar{\lambda}>\lambda>(1-\mu / C) \bar{\lambda}$, then $u(x)>u\left(x^{\lambda}\right)$ for $|x|$ large with $x_{1}=0$. This is a contradiction and Step 3 is established.

Step 4. If $K$ 三 constant, then $u$ is radially symmetric about the origin and $u^{\prime}(r)<0$ for $r=|x|>0$.

Step 3 implies that $u(x)>u\left(x^{\lambda}\right)$ for all $x \in \boldsymbol{R}^{n}$ with $x_{1}<\lambda$ and for all $\lambda>0$. Letting $\lambda \rightarrow 0$ we obtain that $u\left(x_{1}, \ldots, x_{n}\right) \geqq u\left(-x_{1}, x_{2}, \ldots, x_{n}\right)$ for $x_{1}<0$. Reversing the $x_{1}$-axis, we conclude that $u$ is symmetric about the hyperplane $x_{1}=0$ and $\partial u / \partial x_{1}<0$ for $x_{1}>0$. Since the equation (5.1) is rotationally invariant, the $x_{1}$-axis may be chosen arbitrarily, and it follows that $u(x)$ is radially symmetric and $u^{\prime}(r)<0$ for $r>0$.

Step 5. If $K \equiv$ constant, then $(\bar{\lambda}, \infty) \subseteq \Lambda$.

The proof is very similar to the first and second paragraphs of Step 3. In fact, it is simpler. For, when $K \equiv$ constant, the equation in both (5.8) and (5.9) now takes the following form

$$
\Delta\left(u(x)-u\left(x^{\lambda}\right)\right)+K \frac{f\left(u(x)-u\left(x^{\lambda}\right)\right)}{u(x)-u\left(x^{\lambda}\right)}\left(u(x)-u\left(x^{\lambda}\right)\right)=0,
$$

which allows Lemma 4.25 to apply no matter $\bar{\lambda} \geqq 0$ or $\bar{\lambda} \leqq 0$.

Step 6. When $K \equiv$ constant, let $z_{1}=\bar{\lambda}$ and define $z_{j}$ similarly by repeating Step 2 with respect to $x_{j}$-axis, $j=2, \ldots, n$. Then $u$ is radially symmetric about the point $z=\left(z_{1}, \ldots, z_{n}\right)$ and $u^{\prime}(r)<0$ for $r=|x-z|>0$.

When $K \equiv$ constant, equation (5.1) is both translation- and rotation-invariant. Thus we first perform a translation so that the point $z$ becomes the new origin and then repeat the arguments in Step 4 to conclude Step 6.

Finally, (5.4) follows from Theorem 3.2. Q.E.D.

Next, we consider the positive limit case.

Theorem 5.16. Suppose that (K1), (K2), (K3), (f1) and (f3) hold with $\tau>2$. Suppose further that $K(r) \geqq C r^{-n}$ near $\infty$ in case $2<\tau \leqq n$. Let $u$ be a bounded positive solution of (5.1) with $\lim \sup _{x \rightarrow \infty} u(x)=C_{0}>0$. Then $u$ must be radially symmetric about the origin and $u^{\prime}(r)<0$ for $r=|x|>0$. Furthermore, the asymptotic behavior of $u$ is given by (3.13) or (3.18) with the remainder term $R$ being radial as well.

Proof. The case that $\tau>n$ can be treated in the same manner as Theorem 5.2 in view of Theorem 3.12. Thus we only consider the case that $2<\tau \leqq n$ here. Following the general procedure in the proof of Theorem 5.2, we define $\Lambda$ by (5.5). To establish Step 1, i.e., $\lambda \in A$ for all $\lambda$ sufficiently large, we also proceed analogously. Since Theorem 2.32 and (2.34) in Part I hold (see Section 2 of [LN1]), we have $u(x)>C_{0}$ for all $x \in \boldsymbol{R}^{n}$. Thus for every $r \geqq 2$, there exists $\lambda(r) \geqq r$ such that $u(x)>u\left(x^{\lambda}\right)$ for all $|x| \leqq r$ and $x_{1}<\lambda$ if $\lambda \geqq \lambda(r)$. On the other hand, for $|x|>r$ with $x_{1}<\lambda$ and $\lambda>0$ we have, from (3.18), 
(4.24), (4.17), (4.18), (4.3) and (4.5), that

$$
u(x)-u\left(x^{\lambda}\right)=N_{f, k+1}(|x|)-N_{f, k+1}\left(\left|x^{\lambda}\right|\right)
$$

$$
\begin{aligned}
& +\left(\frac{1}{|x|^{n-2}}-\frac{1}{\left|x^{\lambda}\right|^{n-2}}\right)\left[c_{k+2}+W\left(\frac{1}{|x|}\right)\right] \\
& +\frac{1}{\left|x^{\lambda}\right|^{n-2}}\left[W\left(\frac{1}{|x|}\right)-W\left(\frac{1}{\left|x^{\lambda}\right|}\right)\right]+\frac{1}{|x|^{n-2}} R\left(\frac{x}{|x|^{2}}\right)-\frac{1}{\left|x^{\lambda}\right|^{n-2}} R\left(\frac{x^{\lambda}}{\left|x^{\lambda}\right|^{2}}\right) \\
\geqq & \frac{C \lambda\left(\lambda-x_{1}\right) \log |x|}{|x|^{n-2}\left|x^{\lambda}\right|\left(|x|+\left|x^{\lambda}\right|\right)}-\frac{C \lambda\left(\lambda-x_{1}\right)}{|x|^{n-2}\left|x^{\lambda}\right|\left(|x|+\left|x^{\lambda}\right|\right)}-\frac{C \lambda\left(\lambda-x_{1}\right)}{|x|^{n-2+\beta}|x|\left(|x|+\left|x^{\lambda}\right|\right)} \\
& -\frac{C \lambda\left(\lambda-x_{1}\right)}{|x|^{n-1}\left|x^{\lambda}\right|\left(|x|+\left|x^{\lambda}\right|\right)}-\frac{C\left(\lambda-x_{1}\right)}{\left|x^{\lambda}\right|^{n}} \\
\geqq & \frac{C \lambda\left(\lambda-x_{1}\right) \log |x|}{|x|^{n-2}\left|x^{\lambda}\right|\left(|x|+\left|x^{\lambda}\right|\right)}\left[1-\frac{C^{\prime}}{\log |x|}-\frac{C^{\prime \prime}}{|x|^{\beta} \log |x|}-\frac{C^{\prime \prime \prime}}{\lambda \log |x|}\right]
\end{aligned}
$$

since $|x|<\left|x^{\lambda}\right|$ and where $\beta$ is a number between 0 and 1 . Now we choose $r$ so large that

$$
1-\frac{C^{\prime}}{\log r}-\frac{C^{\prime \prime}}{r^{\beta} \log r}-\frac{C^{\prime \prime \prime}}{r \log r} \geqq \frac{1}{2} .
$$

Then $u(x)>u\left(x^{\lambda}\right)$ for all $|x|>r$ with $x_{1}<\lambda$ and $\lambda \geqq \lambda(r) \geqq r$. Thus $[\lambda(r), \infty) \subseteq \Lambda$.

Next we assert that $\Lambda \cap(0, \infty)$ is open. Suppose that this were false; i.e., that there exist a sequence $\left\{\lambda_{i}\right\}$ and a sequence of points $\left\{x^{i}\right\}$ such that $\lambda_{i} \rightarrow \bar{\lambda} \epsilon$ $\Lambda \cap(0, \infty)$ and $u\left(x^{i}\right) \leqq u\left(x^{i \lambda_{i}}\right)$ with $x_{1}^{i}<\lambda_{i}$. If any subsequence of $\left\{x^{i}\right\}$ converges, say to $\bar{x}$, then as in the proof of Theorem 5.2 we apply Lemma 4.25 to $u(x)-u\left(x^{\bar{\lambda}}\right)$ in the region $x_{1}<\bar{\lambda}$ to reach a contradiction. Therefore we must have that $x^{i} \rightarrow \infty$ as $i \rightarrow \infty$. But then (5.17) implies that $u\left(x^{i}\right)>u\left(x^{i \lambda_{i}}\right)$ for all $i$ sufficiently large since $1>C^{\prime}\left(\log \left|x^{i}\right|\right)^{-1}+C^{\prime \prime}\left|x^{i}\right|^{-\beta}\left(\log \left|x^{i}\right|\right)^{-1}+$ $C^{\prime \prime \prime}\left(\lambda_{i} \log \left|x^{i}\right|\right)^{-1}$ for $i$ large. This is again a contradiction and our assertion is established.

It then follows from exactly the same arguments (since $K$ 丰 constant) as in the proof of Theorem 5.2 (Step 3) that $\Lambda \cap(0, \infty)=(0, \infty)$, i.e., $u(x)>u\left(x^{\lambda}\right)$ for all $x \in \boldsymbol{R}^{n}$ with $x_{1}<\lambda$ and for all $\lambda>0$. Finally we conclude our proof by the same arguments used in Step 4 of the proof of Theorem 5.2. Q.E.D.

It is obvious that Theorem 2 follows from Theorem 5.2 while Theorem 1 follows from Theorem 5.16 and from Theorem 1.4 in [LN1].

Remark 5.18. The results in Sections 3 and 5 for equation (3.1) (or (5.1)) also hold for the more general equation

$$
\Delta u+g(|x|, u)=0
$$


in $\boldsymbol{R}^{n}, n \geqq 3$, where $g$ satisfies the following conditions which are analogous to $(\mathrm{K} 1)-(\mathrm{K} 3)$ and $(\mathrm{f} 1)-(\mathrm{f} 3)$.

(g1) $g(|x|, u)>0$ for all $x \in \boldsymbol{R}^{n}$ and for all $u>0$, and there exists $\tau, p \geqq 0$ such that

$$
g(|x|, u) \leqq C(1+|x|)^{-\tau} u^{p}
$$

for all $x \in \boldsymbol{R}^{n}$, and for $u$ near 0 .

(g2) $g(|x|, u)$ is Hölder continuous in $x$ and is Lipschitz continuous in $u \geqq 0$.

(g3) $g(r, u)$ is strictly decreasing in $r>0$ (for every $u>0$ fixed).

(g4) For every compact subset $S$ of $[0, \infty)$ there exists a constant $C>0$ such that

$$
|g(|x|, u)-g(|y|, u)| \leqq C|| x|-| y||\left[|x|^{-\tau}|y|^{-1}+|x|^{-1}|y|^{-\tau}\right] u^{p}
$$

for every $u \in S$ and for all $x, y \in \mathbb{R}^{n}$ with $|x|,|y|$ large.

(g5) There exists a constant $C>0$ such that

$$
|g(|x|, u)-g(|x|, v)| \leqq C|u-v|\left(|u|^{p-1}+|v|^{p-1}\right)(1+|x|)^{-\tau}
$$

for every $x \in \boldsymbol{R}^{n}$ and for all $u, v$ small and positive.

We do not repeat the statements or the proofs of those results because they are very similar to the results in Sections 3 and 5. We point out that the strict monotonicity assumed in (g3) is necessary as the following example shows.

Example 5.20. Let $u_{0}$ be a fixed classical radial positive solution of

$$
\Delta u+u^{\frac{n+2}{n-2}}=0
$$

in $\boldsymbol{R}^{n}, n \geqq 3$; for instance,

$$
u_{0}(r)=\left(\frac{\sqrt{n(n-2}}{1+r^{2}}\right)^{\frac{n-2}{2}}, \quad r>0 .
$$

Define

$$
g(r, u)= \begin{cases}u^{\frac{n+2}{n-2}} & \text { if } u \leqq u_{0}\left((r-1)_{+}\right), \\ {\left[u_{0}\left((r-1)_{+}\right)\right]^{\frac{n+2}{n-2}}} & \text { if } u>u_{0}\left((r-1)_{+}\right),\end{cases}
$$

where $(r-1)_{+}=\max \{r-1,0\}$. Then obviously $u(x)=u_{0}(|x-y|)$ is a solution of $\Delta u+g(|x|, u)=0$ in $\boldsymbol{R}^{n}$ for every $|y| \leqq 1$, and $g$ satisfies (g1) - (g5) except the strict monotonicity in (g3). (Note that $g$ is in fact, monotonically nonincreasing in $r>0$.) It is also clear that we may replace $u^{\frac{n+2}{n-2}}$ above by other nonlinearities.

\section{Concluding Remarks}

A few remarks concerning the various hypotheses and consequences of our results are in order. 
Remark 6.1. Obviously there must be some decay conditions near $\infty$ imposed on solutions $u$ in order to derive the radial symmetry of $u$. (It is easy to construct counterexamples otherwise.) However, it is still natural to ask how restrictive the decay condition in Theorem 5.2 is. First, observe that (5.3) and the condition on $p$ in Theorem 5.2 substantially improve that of Theorems $1^{\prime}$ and 1 " of [GNN; p. 378 and p. 380 , respectively]. Then we notice that if $K(r) \sim r^{-\tau}$ near $\infty$ for some $0<\tau \leqq 2$, Theorem 3.35 in [N] guarantees that all positive solutions $u$ of (5.1) with $f(u)=u^{p}, p>1$ must satisfy

$$
\bar{u}(r) \leqq \begin{cases}C r^{\frac{\tau-2}{p-1}} & \text { if } 0<\tau<2, \\ C(\log r)^{-\frac{1}{p-1}} & \text { if } \tau=2,\end{cases}
$$

where $r=|x|$ and $\bar{u}(r)$ is the average of $u$ on the sphere $|x|=r$. (Compare (6.2) and (5.3).)

As for the condition imposed on solutions near $\infty$ in Theorem 5.16, it seems very minimal.

Remark 6.3. Comparing our symmetry results to the previous ones in [GNN, e.g., Theorem 1 "l, we see that the monotonicity assumption of $g(r, u)$ in $u>0$ is now removed in both Theorems 5.2 and 5.16 (see Remark 5.18 also). The key new ingredients here are Lemmas 4.6 and 4.9, and of course Theorem 3.2. We should point out that the Hopf Boundary Point Lemma at $\infty$ for the halfspace obtained in [GNN; Lemma $\left(H_{1}^{\prime \prime}\right)$ ] also plays a crucial role in our estimates.

Remark 6.4. Combining Theorem 2 above, Theorem 2.1 in [LN2] and a recent result of YANAGIDA [Y], we see that the Matukuma equation (1.2) with $1<p<\frac{n+2}{n-2}$ possesses exactly one solution with finite total mass; moreover, it is radial. This fills in the gap $1<p<\frac{n-1}{n-2}$ left open in [LN2].

Finally, we mention that other related recent papers include [BN1,2], [CGS] and $[C L]$.

Note added in proof. In a very recent paper of the first author, YI LI, "On the positive solutions of Matukuma equation", it is proved that all positive entire solutions of equation (1.2) (without any conditions imposed on such solutions) are radially symmetric about the origin. Thus, a complete understanding of all positive solutions of the Matukuma equation is achieved.

Acknowledgment. This research was supported in part by the National Science Foundation. 


\section{References}

[BN1] H. BeRESTYCKI \& L. NiRENBERG, Monotonicty, symmetry and anti-symmetry of solutions of semilinear elliptic equations, $J$. Geometry Physics 5 (1988), $237-275$.

[BN2] H. BerESTYCKI \& L. NIRENBERG, Some qualitative properties of solutions of semilinear elliptic equations in cylindrical domains, Analysis, et Cetera: Research papers published in honor of Jürgen Moser (P. H. RABINOwITZ \& E. ZEHNDER, Eds.), Academic Press, 1990, 115-164.

[CGS] L. Caffareldi, B. Gidas \& J. Spruck, Asymptotic symmetry and local behavior of semilinear elliptic equations with critical Sobolev growth, Comm. Pure Appl. Math. 42 (1989), 271-297.

[CL] W. CHEN \& C. LI, Classification of solutions of some nonlinear elliptic equations, Duke Math. J. 63 (1991), 615-622.

[FL] B. FRANCHI \& E. LANCONELLI, Radial symmetry of the ground states for a class of quasilinear elliptic equations, Nonlinear Diffusion Equations and Their Equilibrium States (W.-M. NI, L. A. Peletier \& J. SERrin, Eds.), Vol. 1 (1988), $287-292$.

[GNN] B. GidAs, W.-M. NI \& L. NiRENBERG, Symmetry of positive solutions of nonlinear elliptic equations in $\boldsymbol{R}^{n}$, Advances in Math. Supplementary Studies 7A (L. NACHBIN Ed.), (1981) 369-402.

[GT] D. Gmbarg \& N. Trudinger, Elliptic Partial Differential Equations of Second Order, 2nd edition, Springer-Verlag, 1983.

[LN1] Y. LI \& W.-M. NI, On conformal scalar curvature equations in $\boldsymbol{R}^{n}$, Duke Math J. 57 (1988), 895-924.

[LN2] Y. LI \& W.-M. NI, On the existence and symmetry properties of finite total mass solutions of the Matukuma equation, the Eddington equation and their generalizations, Arch. Rational Mech. Anal. 108 (1989), 175-194.

[N] W.-M. NI, On the elliptic equation $\Delta u+K(x) u^{\frac{n+2}{n-2}}=0$, its generalizations and applications in geometry, Indiana Univ. Math. J. 31 (1982), 493-529.

[Y] E. YANAGDA, Structure of positive radial solutions of Matukuma's equation, Japan J. Indust. Appl. Math. 8 (1991), 165-173. 